\title{
THE LINEARLY POLARIZED LIGHT FIELD IN CLEAR, TROPICAL MARINE WATERS: SPATIAL AND TEMPORAL VARIATION OF LIGHT INTENSITY, DEGREE OF POLARIZATION AND e-VECTOR ANGLE
}

\author{
THOMAS W. CRONIN ${ }^{1, *}$ AND NADAV SHASHAR ${ }^{2}$ \\ ${ }^{1}$ Department of Biological Sciences, University of Maryland Baltimore County, 1000 Hilltop Circle, Baltimore, MD \\ 21250, USA and ${ }^{2}$ The Interuniversity Institute of Eilat, PO Box 469, Eilat 88103, Israel \\ *e-mail: cronin@umbc.edu
}

Accepted 19 April 2001

\begin{abstract}
Summary
Sensitivity to polarized light is widespread among marine animals, including crustaceans, cephalopods and some fishes. They use this ability to orient and find prey, and possibly for a number of other visual tasks. Unlike the ultraviolet-sensitive polarization receptors of most insects, the polarization receptors of marine invertebrates tend to be maximally sensitive near $500 \mathrm{~nm}$, suggesting that polarized light in water differs from that in air. The underwater field of partially linearly polarized light has been studied for nearly $\mathbf{5 0}$ years, but data are still limited and sparse. We measured the submarine polarized light field from 350 to $600 \mathrm{~nm}$ throughout the day on a coral

along any given line of sight at this depth is a relatively simple function of solar position and that the degree of polarization is greatest $60-90{ }^{\circ}$ from the sun. Both e-vector angle and degree of polarization vary only slightly with wavelength, although light is sometimes less polarized in the ultraviolet. Since light is most intense at medium wavelengths and polarization is nearly maximal at these wavelengths, invertebrate polarization photoreceptors are spectrally well placed. Also, the relative spectral constancy of the angle and degree of polarization supports fish polarization sensitivity, which relies on spectrally diverse photoreceptor sets.
\end{abstract} reef in the Florida Keys at a depth of $15 \mathrm{~m}$ using the underwater laboratory Aquarius as a research platform. Our results show that the angle of polarization as viewed

Key words: polarization, polarized light, vision, underwater light, evector angle.

\section{Introduction}

Light coming from the sun is essentially unpolarized. Despite this, natural light fields normally contain a complex pattern of partially linearly polarized light, produced mainly by reflection from dielectric surfaces (such as water or waxy cuticles) or by scattering in the air and water. This pattern, or aspects of it, can be visualized by most animal species, including the great majority of mobile marine invertebrates (for a review, see Waterman, 1981). Many species of marine fishes also respond to features of the polarized light field (Hawryshyn, 1992; Hawryshyn, 2000).

Marine animals may use their ability to perceive the submarine light field for orientation (e.g. Hawryshyn and Bolger, 1990; Goddard and Forward, 1991) or for enhancing the visibility of prey (Shashar et al., 1998; Shashar et al., 2000) and possibly also to increase visual contrast (Lythgoe and Hemmings, 1976; Shashar and Cronin, 1996) or to improve intraspecific communication (Shashar et al., 1996; Marshall et al., 1999). As is the case with insects operating in terrestrial environments, marine invertebrates generally process polarized light using two sets of photoreceptors having orthogonally arrayed microvilli (Goldsmith, 1977; Saidel et al.,
1983; Waterman, 1981). The fishes are somewhat different, in that the mechanism used for polarization analysis is not well understood, but it evidently involves several distinct cone classes (Hawryshyn and McFarland, 1987; for reviews, see Hawryshyn, 1992; Hawryshyn, 2000).

Of the marine invertebrates, polarization sensitivity is best studied in the cephalopods and crustaceans. However, these animals differ strikingly from their terrestrial counterparts in the spectral region to which the polarization receptors are maximally sensitive. While the polarization receptors of insects operate primarily in the ultraviolet (and those viewing the celestial hemisphere are almost exclusively ultraviolet classes; see Waterman, 1981; Rossel, 1989), those of crustaceans and cephalopods are almost invariably middlewavelength types, being maximally sensitive to light near $500 \mathrm{~nm}$. In fact, among marine crustaceans, only some species of stomatopod have ultraviolet-sensitive photoreceptors that are also capable of polarization analysis (Cronin et al., 1994b), and no known cephalopod has any photoreceptor class with sensitivity peaking in the ultraviolet (Messenger, 1981). The situation in stomatopods is particularly interesting. These 
animals have up to 16 different visual pigments, homologues of which may vary substantially in their spectral placement among closely related species (Cronin et al., 1994a; Cronin et al., 1996; Cronin et al., 2000). Despite this, the two sets of photoreceptors devoted specifically to polarization analysis are highly conserved among stomatopod species. In most cases, their peak sensitivities are confined to two narrow spectral ranges, one near $360 \mathrm{~nm}$ and the other between 495 and $505 \mathrm{~nm}$ (Cronin et al., 1994b; Cronin et al., 2000).

What differences between the polarization properties of marine and terrestrial environments explain the divergent evolutionary paths taken by the visual systems of their invertebrate inhabitants? In an analysis of insect polarization sensitivity, Seliger et al. (Seliger et al., 1994) concluded that, under conditions in which polarization from the sky is diminished by atmospheric haze, the best polarization signal should exist in the ultraviolet. However, it is not obvious that the same should hold true under water, for two reasons. First, because of absorption and scattering, the intensity of ultraviolet light at moderate depth is relatively low (compared with the terrestrial case), even in quite clear water. Also, the much greater scattering of ultraviolet wavelengths in water should rapidly remove any polarization signal, even at short ranges. In pioneering measurements, Ivanoff and Waterman (Ivanoff and Waterman, 1958) showed that the degree of polarization of light in water increases steadily with wavelength above $450 \mathrm{~nm}$ (their instrument was incapable of analysis in the ultraviolet). If this is generally true, the evolution of polarization receptors specializing in middle to long wavelengths may be favored.

At present, the overall characteristics of the polarization light field in water are poorly understood. Extensive modeling of this field suggests how it might appear from just beneath a completely flat water surface (Horváth and Varjú, 1995), but only a single study has described the actual spatial distribution of linear polarization in any detail (Waterman, 1954). The data of Waterman were obtained by eye at a very shallow depth $(2-3 \mathrm{~m})$ using a hand-held polarization axis finder. Submersible polarimeters with scanning or filtered optics have been used to collect a diversity of data in shallow to deep marine waters (e.g. Ivanoff and Waterman, 1958; Tyler, 1963; for a review, see Jerlov, 1976), but constraints on the spectral coverage or the rate of data collection have limited the range of results obtained by these instruments, particularly regarding the spatial distribution of submarine polarized light. The development of polarization camera sensors (e.g. Wolff and Andreou, 1995; Shashar et al., 1995) has provided an efficient means of acquiring spatial images of the polarized light field, but with very little spectral information.

We therefore set out to investigate the polarized light field in natural marine waters, sampling the spectrum of partially linearly polarized light throughout the overhead light field throughout the day. Our results provide the first description of the polarized light field over a broad spectral range at moderate depths in the ocean and may help explain the evolution of particular spectral classes of polarization photoreceptors in the eyes of marine animals.

\section{Materials and methods}

Field work was completed in August 1999, during a mission to the Aquarius underwater laboratory, located at a depth of $15 \mathrm{~m}$ on Conch Reef off Key Largo, Florida, USA; latitude $27^{\circ} 50^{\prime} 00^{\prime \prime} \mathrm{N}$, longitude $80^{\circ} 27^{\prime} 13^{\prime \prime} \mathrm{W}$. The National Undersea Research Center, Florida Keys Program, provided all logistical support. Flat platforms attached to the laboratory, also at $15 \mathrm{~m}$ depth (and approximately $3 \mathrm{~m}$ above the seabed), were used to support and stabilize the polarimeter. During the period when polarization measurements were being taken, the optical quality of the water was moderate, with maximum visibility limited to approximately $25 \mathrm{~m}$. Although not directly observable from the underwater collection site, the skies over the underwater habitat were partly cloudy on days when measurements were taken.

All data were obtained using a submersible polarimeter based on an Ocean Optics Inc. high-sensitivity S1000 spectrometer with enhanced ultraviolet sensitivity. This spectrometer was calibrated for wavelength using the emission lines of a mercury lamp and for spectral sensitivity using a calibrated spectral lamp. The spectrometer itself and the computer to which it was attached were operated from within the laboratory, while the polarimeter head was mounted on an adjustable tripod placed on one of the external platforms. The head contained a sheet polarizer (Polaroid HNP'B, which is effective in both visible and ultraviolet spectral regions) mounted on a shaft that could be rotated under computer control and placed in front of a radiometric aperture that collected light over a solid angle of $15^{\circ}$ (under water). Light entering this collector, having passed through the linear polarizer, was conducted to the spectrometer by a $10 \mathrm{~m}$ optical fiber, $600 \mu \mathrm{m}$ in diameter. In this instrument, spectral bandwidth is determined by fiber diameter, so spectral resolution was low, with a bandwidth at half-maximum of approximately $40 \mathrm{~nm}$.

To collect the spectrum of partially linearly polarized light, the polarimeter head was first adjusted by a diver to the desired azimuth angle and elevation using a compass and inclinometer. Data were then obtained automatically, in the following sequence. First, the polarizer was rotated to transmit the horizontal e-vector, and the spectrometer adjusted itself under computer control for a proper exposure. Next, the sheet of polarizer was rotated so that an opaque section covered the radiometric collector, and three successive spectra of dark noise were obtained, averaged and stored by the operating computer. The polarizer was then rotated rapidly, under computer control, to three orientations of its transmitted evector, $0^{\circ}, 45^{\circ}$ and $90^{\circ}$. This sequence was repeated a total of three times, and the average spectrum of light at each orientation was stored. The entire operation was completed in approximately $30 \mathrm{~s}$, and the polarimeter head was then adjusted to the next desired orientation. 
Data were collected at increments of $45^{\circ}$ over $360^{\circ}$ of azimuth and at $30^{\circ}$ increments over $90^{\circ}$ of elevation; only the upper hemisphere of the light field was sampled. Since the underwater laboratory itself was adjacent to the platforms from which measurements were taken, the polarimeter was moved from one side of the laboratory to the other half-way through the measurement series to avoid any influence of its presence on the data. Because of the need to readjust the polarimeter to each new orientation and to move it from one location to the other once during each series, up to $2 \mathrm{~h}$ was required to complete a measurement series at 25 orientations. To minimize this time, the diver immediately returned to the hatch of the habitat after making each adjustment and waited to be alerted to move to the next setting. At some orientations of the polarimeter, the light intensity was sufficiently bright to saturate the spectrometer, so the intensity was reduced using a sheet of black nylon mesh placed over the entrance to the polarimeter. Preliminary tests demonstrated that this material did not disturb the polarization signal, and its spectral attenuation was included in analyses of scans taken when it was present.

All data were stored for analysis, which followed the Aquarius mission. For each time and polarimeter orientation, the spectra obtained at each of the three orientations of the polarizer (each of which was an average of three raw spectra) were dark-corrected using the dark spectrum obtained simultaneously, and then smoothed using a running $5 \mathrm{~nm}$ average. The smoothed spectra were then analyzed at $1 \mathrm{~nm}$ intervals for overall intensity, degree of polarization and evector angle, from 350 to $600 \mathrm{~nm}$ (the actual data were collected from 300 to $900 \mathrm{~nm}$, but at the depth at which measurements were made, light was insufficient for polarization analysis outside the range $350-600 \mathrm{~nm}$ ). (For a description of the mathematics required to analyze polarization data such as these, see Wolff and Andreou, 1995.)

\section{Results}

Each primary data set consists of three smoothed spectra (each an average of three measurements) from which the dark noise spectrum has been subtracted. Fig. 1A shows an example of one such set, acquired at 09:01 h Eastern Daylight Time (EDT) on 16 August 1999 looking North $\left(0^{\circ}\right)$ at an elevation of $60^{\circ}$ above the horizontal. In this figure, the blue line shows the uncorrected spectrum for an e-vector orientation of $0^{\circ}$ (horizontal), the red line is that at $90^{\circ}$ (vertical), and the gray line shows the spectrum for the $45^{\circ}$ e-vector orientation. From these spectra, the three primary attributes of partial linear polarization can readily be computed at $1 \mathrm{~nm}$ intervals, and these are illustrated in Fig. 1B. In this particular instance, e-vector angle was quite constant at approximately $60^{\circ}$, while the degree of polarization was rather low, dropping from approximately $20 \%$ at $350 \mathrm{~nm}$ to near $10 \%$ at $600 \mathrm{~nm}$.

An entire data series, showing the polarized light field throughout the upper hemisphere, includes 25 sets of polarization measurements (eight each at $0^{\circ}, 30^{\circ}$ and $60^{\circ}$ of elevation and an additional one at $90^{\circ}$ ). An example of one such data set is provided in Fig. 2, which shows the results of a measurement series conducted between 12:16 and 13:22 h EDT on 16 August 1999. At this time, the sun was nearly overhead throughout the time data were collected, thus producing a relatively simple polarization field with nearly horizontal e-vectors at all locations and wavelengths, and a gradually decreasing degree of polarization with increasing elevation. Note that, throughout the entire upward hemisphere, there is at most only slight spectral variation at any given orientation, either in degree of polarization or in evector angle. However, two minor maxima in percentage polarization are sometimes observable, at approximately 400 and $560 \mathrm{~nm}$.

An alternative view of the polarization light field at midday is presented in Fig. 3, which provides a schematic
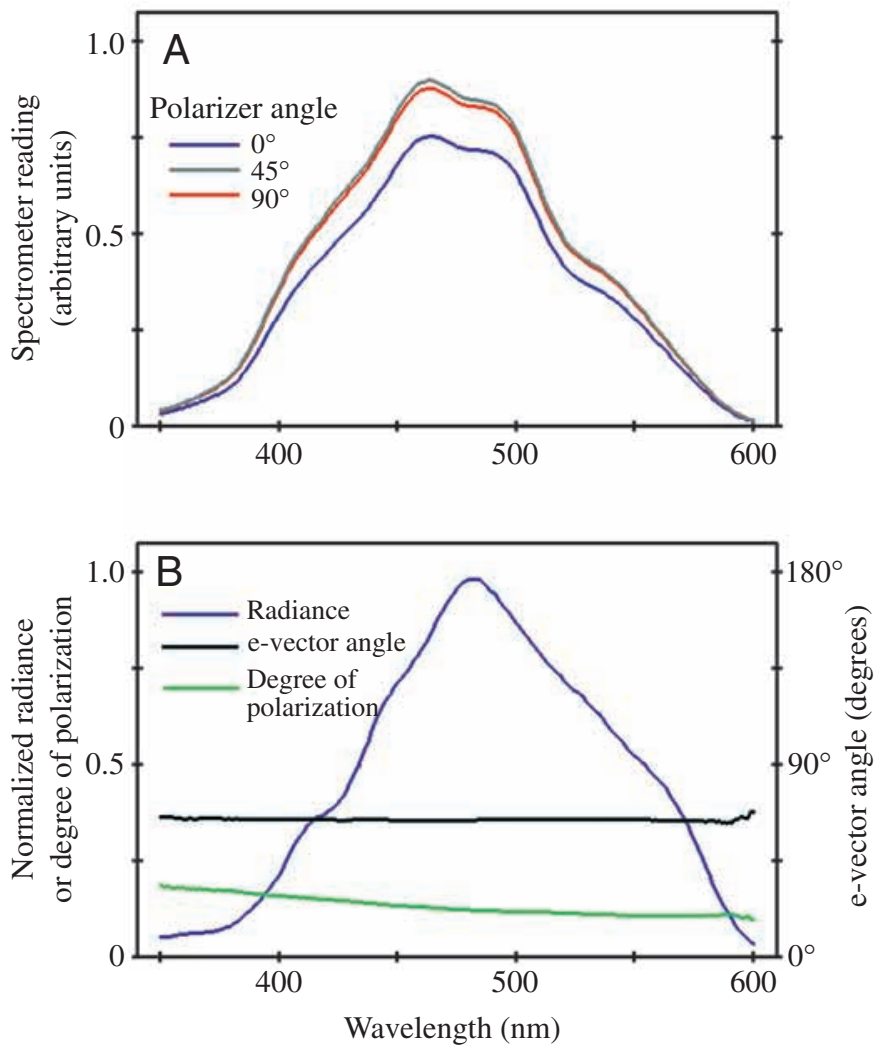

Fig. 1. Analysis of polarization at one direction of view. Data were collected at 09:01 h Eastern Daylight Time on 16 August 1999, with the polarimeter looking North at $60^{\circ}$ elevation. (A) Averaged, uncorrected spectra of intensity at three orientations of the polarization analyzer mounted in front of the radiance collector. Blue line, analyzer at $0^{\circ}$ (horizontal); gray line, analyzer at $45^{\circ}$; red line, analyzer at $90^{\circ}$. (B) Spectra of partial, linear polarization obtained by analyzing the data in A. Blue line, normalized radiance (corrected for spectrometer spectral response; the curve is normalized to $5.24 \times 10^{11}$ photons $\mathrm{cm}^{-2}$ sterad $^{-1} \mathrm{~nm}^{-1} \mathrm{~s}^{-1}$ ); black line, e-vector angle $\left(0^{\circ}\right.$ is horizontal, with angles increasing counterclockwise; righthand axis); green line, degree of polarization (1.0 is fully polarized light). 


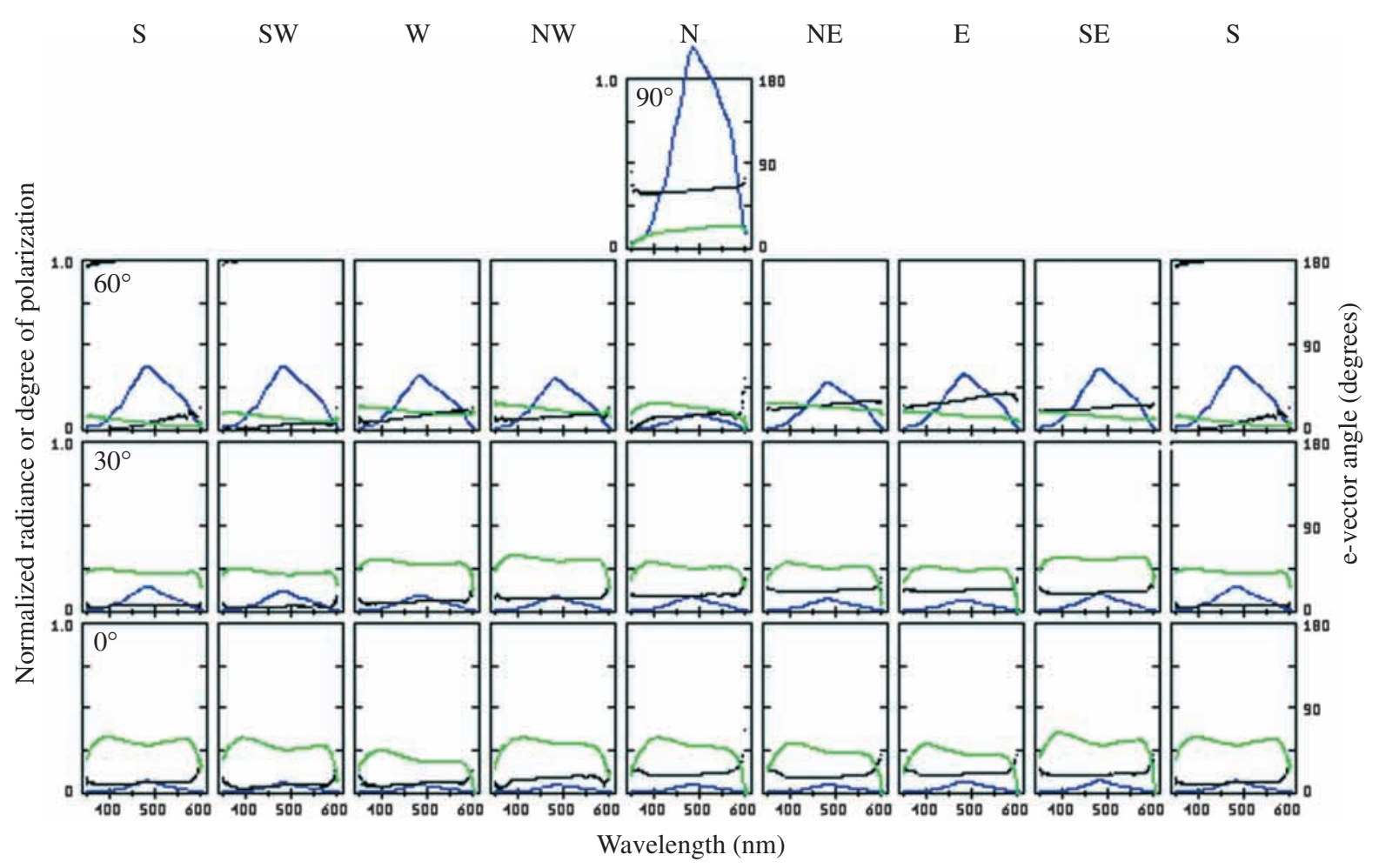

Fig. 2. The polarization light field near midday; data were obtained between 12:16 and 13:22 h Eastern Daylight Time on 16 August 1999. Each panel shows the spectra of radiance, degree of polarization and e-vector angle at one orientation of the polarimeter, indicated by the compass directions at the top and elevations $\left(0-90^{\circ}\right)$ in the panels on the left. Blue lines, radiance (all curves are scaled such that 1.0 on the left-hand ordinate is equivalent to $2.92 \times 10^{12}$ photons $\left.\mathrm{cm}^{-2} \mathrm{sterad}^{-1} \mathrm{~nm}^{-1} \mathrm{~s}^{-1}\right)$; black lines, e-vector angle $\left(0^{\circ}\right.$ is horizontal, with angles increasing counterclockwise; right-hand ordinate); green lines, degree of polarization (left-hand ordinate). Note that data for South are plotted twice, at each end of each row, to provide continuity.

Fig. 3. Representations of the polarized light field near midday at four wavelengths, obtained between 12:16 and 13:22 h Eastern Daylight Time on 16 August 1999 (same data as illustrated in Fig. 2). Each part of the figure represents the polarization pattern in the upper hemisphere of view at the wavelength indicated, as seen by an observer looking upwards (note that East and West are reversed from their locations on a standard compass because of this unusual direction of view). The outer circle represents the horizontal, with the inner circles representing elevations of $30^{\circ}$ and $60^{\circ}$. The lighter inner region of the figure represents the theoretical location of Snell's window (the angular extent of the sky above the water's surface after refraction at the air/water interface), although this was not clearly seen at the measurement depth of $15 \mathrm{~m}$. At each location where the spectrum of partial linear polarization was measured and analyzed (as in Fig. 1), the e-vector angle is indicated by the tilt of the line relative to a tangent to the circle passing through that point, and the percentage polarization is indicated by the thickness of the line (key provided at lower right). The approximate location of the sun midway through the measurement series is indicated by the sun symbol within Snell's window.

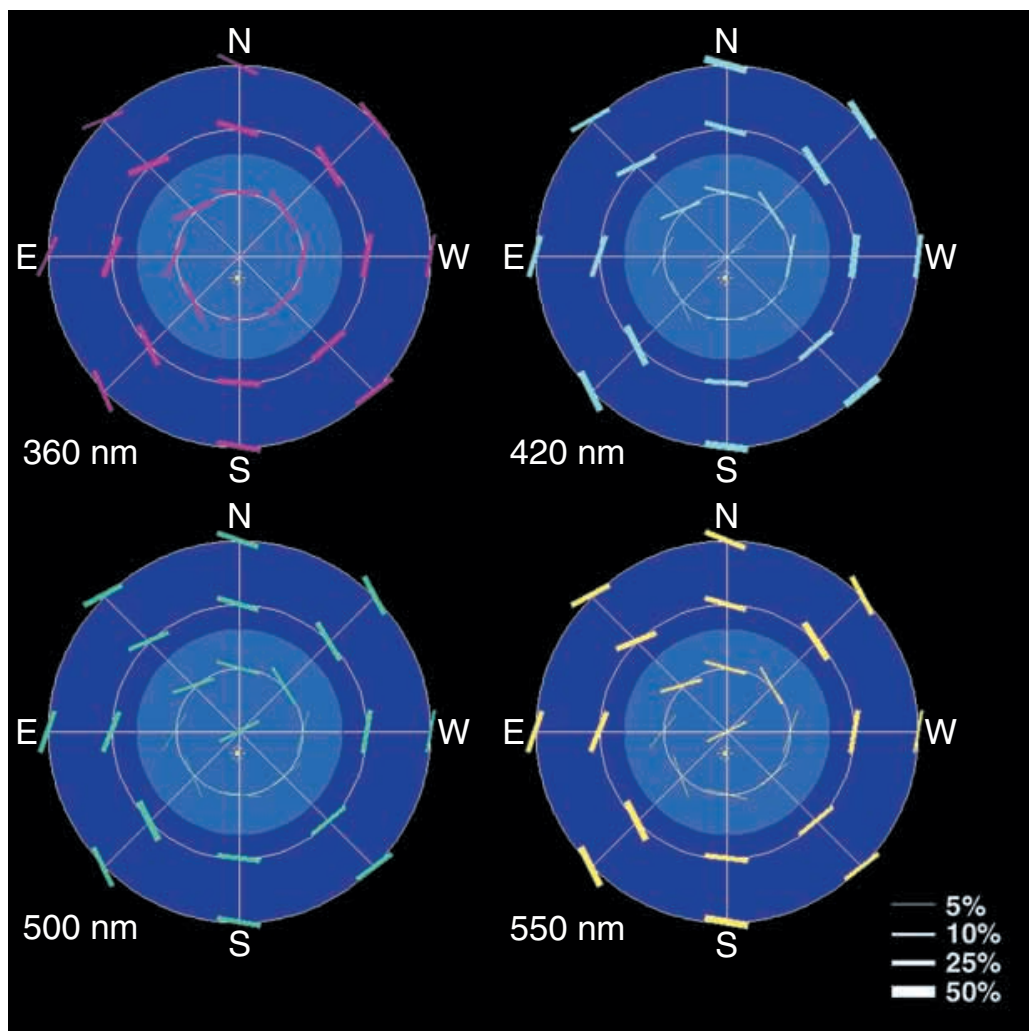


representation of the distribution of polarization in the overhead hemisphere for the same series of data as that of Fig. 2. In Fig. 3 (and also in Figs 4 and 5), the circles indicate the horizontal plane and elevations of $30^{\circ}$ and $60^{\circ}$. The brighter central region represents the extent of Snell's window, where light from the celestial hemisphere would be seen from under water (at the location from which our polarization measurements were made, no sharp border was visible at the margins of Snell's window, but the figures suggest the general location of this region of somewhat brighter light). The cardinal points for East and West are reversed in this depiction from those of a standard compass because the view is upwards, and therefore East is seen to the left of North. The approximate position of the sun midway through the measurement series is indicated as well. The pattern of polarization is illustrated at four wavelengths, and at each point where a polarization measurement was taken, a tilted line indicates the e-vector angle (relative to the horizontal) at that location. The thickness of the line increases with the degree of polarization (see the key in the lower right corner of the plot for interpretation). As in the series depicted in Fig. 2, polarization is maximal near the horizon and is nearly horizontal at all locations. The distribution of polarization at all wavelengths is similar, although the degree of polarization is typically slightly less at $360 \mathrm{~nm}$ than at longer wavelengths.

Figs 4 and 5 use similar conventions to those of Fig. 3, but show the polarization field in midmorning (Fig. 4, 09:23-11:42 h EDT, 15 August 1999) and near sunset (Fig. 5, 17:51-19:21 h EDT, 15 August 1999). At these times, the rays from the sun travel somewhat laterally, producing tilted evector orientations and a rather complicated distribution of the degree of polarization. Polarization tends to achieve its maximum at angles approximately $60-90^{\circ}$ from the sun, and thus the light reaches degrees of polarization near 40-50\% well above the horizontal line of sight. In general, the angle of polarization is roughly perpendicular to the direction of the sun's rays, as in the atmosphere. Note that, at many locations, light in the ultraviolet is slightly less polarized than at longer wavelengths, unlike the typical situation in the atmosphere. Also note that there is no remnant of the atmospheric polarization pattern in the underwater pattern at this depth; the pattern is not obviously different within Snell's window compared with the overall distribution of polarization. The loss of the atmospheric pattern must be due to the destruction of the original polarization by multiple-path scattering in the water column.

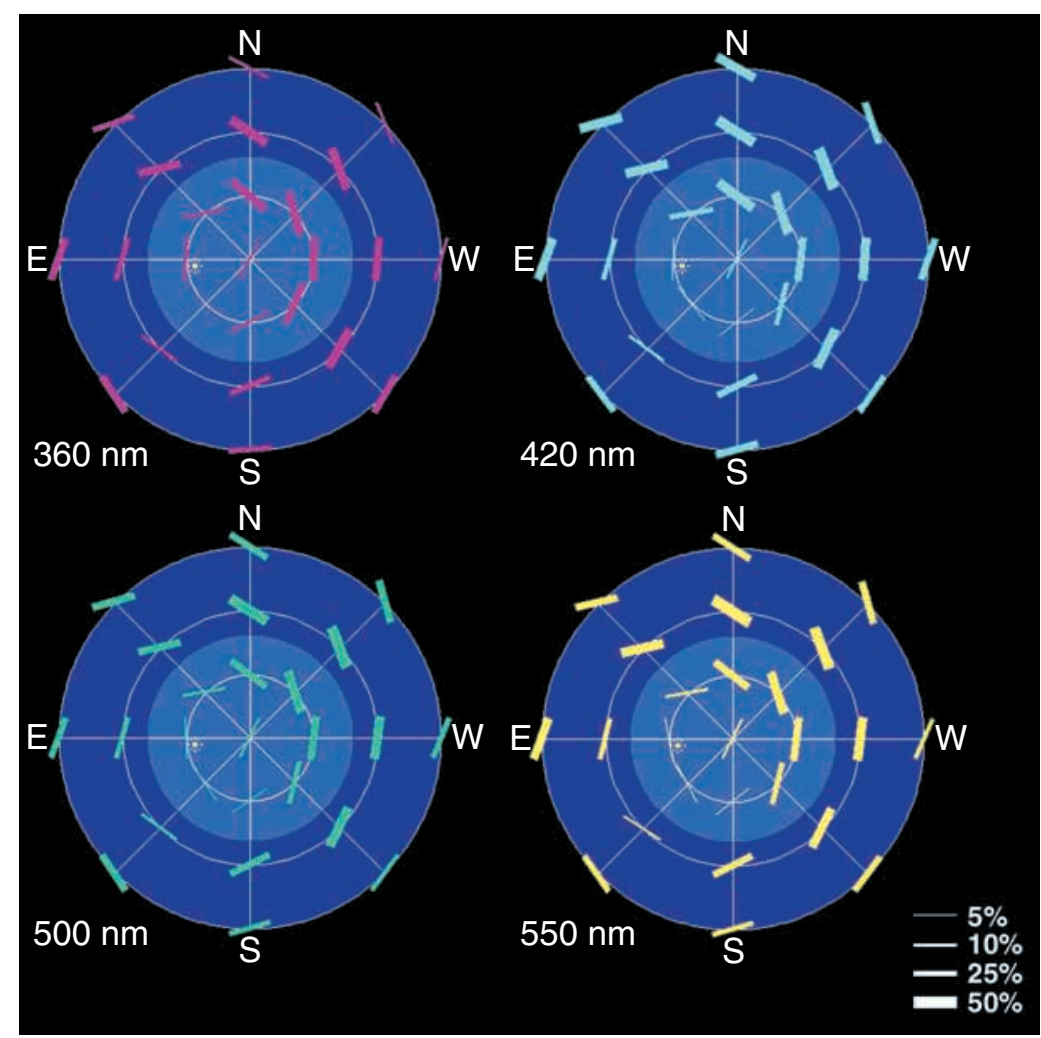

Fig. 4. Representations of the polarized light field at mid-morning at four wavelengths, obtained on 15 August 1999 between 09:23 and 11:42 h Eastern Daylight Time. Otherwise as in Fig. 3.

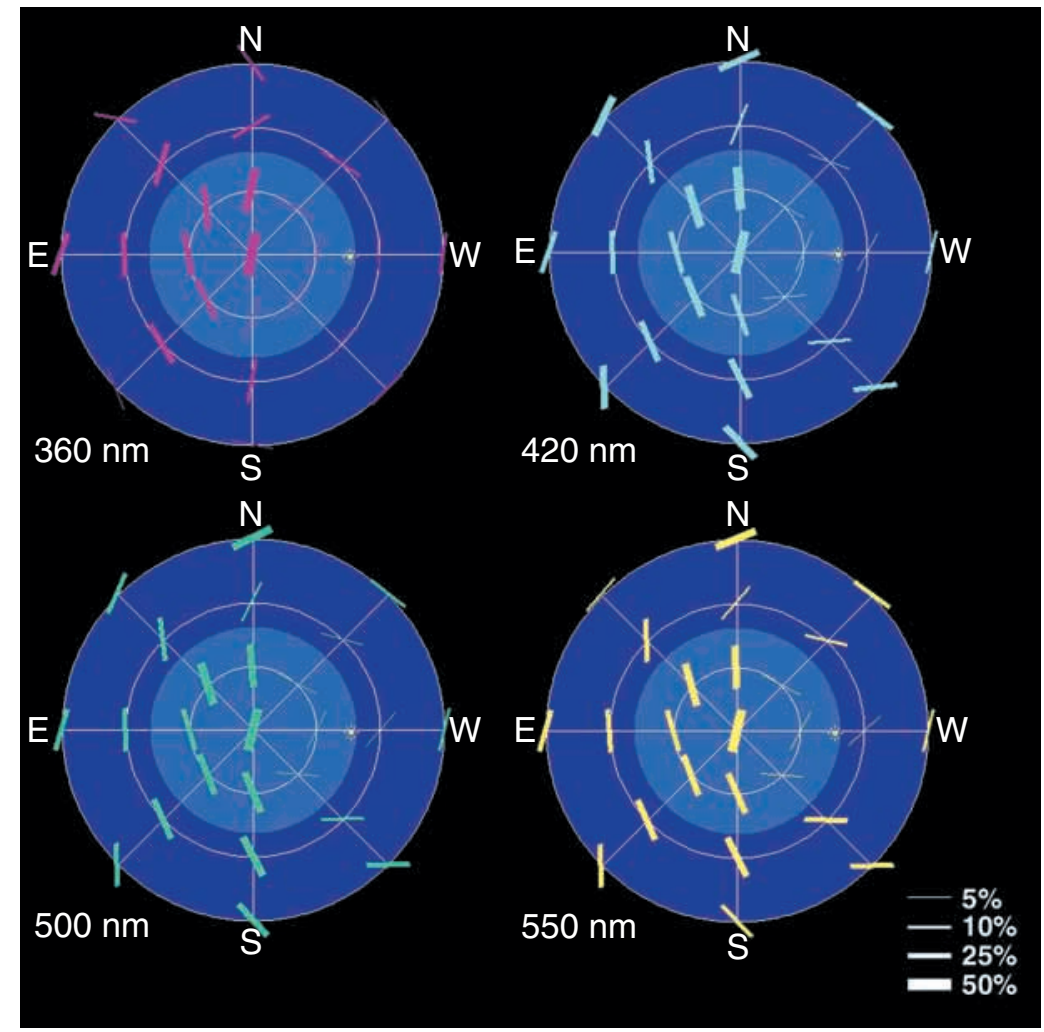

Fig. 5. Representations of the polarized light field near sunset at four wavelengths, obtained on 15 August 1999 between 17:51 and 19:21 h Eastern Daylight Time. Otherwise as in Fig. 3. 


\section{Discussion}

The data presented here are the first detailed, quantitative description of the spectral distribution of polarized light in water. As such, they provide a new view of the pattern of polarization in nature and encourage speculation about the evolution of polarized-light sensitivity and polarization vision in the sea. Nevertheless, this data set is limited in some regards, and it may be helpful to consider these limitations first.

Data were collected on a total of only five occasions over 2 days; the complexity of the data collection process and the necessity to maintain clear communication between a diver in the water and the computer operator in the underwater habitat constrained the potential quantity of data that could be obtained. This was true despite the fact that, on one of the days (when three of the five series had been completed), the diver orienting the polarimeter established an unofficial record for the time spent underwater on a single day of any Aquarius mission! Furthermore, since the polarimeter was mounted over a platform, only the upper hemisphere of the light field could be sampled. In future measurements of the polarization field, both a far more efficient means of moving the instrument and a way to obtain full spherical field coverage are highly desirable. Despite the low time resolution, the polarization patterns that we measured (see Figs 3-5) are roughly symmetrical about the plane including the sun, which indicates that, over the course of each measurement set, there was little overall change in the pattern. However, because there were clouds in the sky overhead (which could not be seen from under water), the light intensity and directionality varied unpredictably throughout each series. Finally, the field was measured at a single depth $(15 \mathrm{~m})$ in moderately clear water. In future work, it would be desirable to sample the polarization field under a variety of water and sky quality conditions and, equally importantly, at a series of depths and in water of varying overall depth. Keeping these caveats in mind, what do our results indicate about the underwater polarization field?

Throughout our work, the degree of polarization never reached values much exceeding $50 \%$, which is lower than commonly reported levels of polarization in the atmosphere (particularly near sunrise and sunset on clear days; see Waterman, 1981). Of course, polarization content varies with water quality as well, and values near $70 \%$ polarization have been recorded in a freshwater lake near twilight (Novales Flamarique and Hawryshyn, 1997). The degree of polarization in water varies with azimuth, and research has consistently revealed that, for horizontal lines of sight, it reaches minimum values along the solar and antisolar axes (for reviews, see Jerlov, 1976; Waterman, 1981). An examination of the entire pattern of polarization (Figs 3-5) shows why this is so; polarization tends to be greatest approximately $60-90^{\circ}$ from the sun, so whenever the sun is not directly overhead, the tilted plane of greatest polarization is intersected on axes $\pm 90^{\circ}$ to the line connecting the observer and the apparent position of the sun.

The relatively low polarization values in water must challenge the polarization vision systems of marine animals.
For instance, behavioral studies of fish orientation in polarized light fields have found that orientation is impossible when polarization falls below $65-75 \%$. Consequently, in natural waters, fish may be able to use natural fields for orientation only rarely, mainly near times of twilight (Novales Flamarique and Hawryshyn, 1997). As a consequence of their microvillar structure, invertebrate photoreceptors inherently have much greater polarization sensitivity than vertebrate types, and it is likely that crustaceans and cephalopods can always make use of submarine polarization throughout the day, at least in some directions of view (Goldsmith, 1977; Waterman, 1981). Tyler (Tyler, 1963) has calculated that, even under cloudy skies, light in deep water can be $30-40 \%$ polarized, which may be visible to marine invertebrates.

The pattern of polarization at $15 \mathrm{~m}$ depth, in moderately clear water, is very reminiscent of the pattern in air for similar positions of the sun (see Waterman, 1981; Wehner and Rossel, 1985; Rossel, 1989). The pattern is concentric around the sun, making it symmetrical about the vertical plane passing through the sun and zenith (see Figs 3-5). Thus, near noon, there is a circle of maximum polarization, with horizontal e-vectors lying in the horizontal plane (e.g. Fig. 3), while at sunrise or sunset, the band of maximum polarization passes nearly overhead in a great circle, with the e-vectors oriented vertically (Fig. 5). The origin of this pattern is the same as for the celestial pattern; photons are preferentially scattered towards an observer when their e-vectors are perpendicular to the plane passing through the observer, the light source and the point of scattering, and scattering is particularly favored at right angles. The pattern is actually more stable in water than in air for a given solar angle, since the light in air can be highly directional in clear skies and nearly omnidirectional under clouds, but underwater at moderate depths, the region of greatest brightness will always be near the refracted position of the sun in the sky, although the distribution of brightness will always be broad due to in-water scattering. Consequently, maximum polarization in water will be reduced compared with that in clear skies in air but greater than under cloudy skies in air, and the overall pattern of polarization is likely to be relatively insensitive to sky state except under heavy overcast (when the pattern should come to resemble that of Fig. 3). In his pioneering work, Waterman (Waterman, 1954) noted that the celestial polarization pattern has little influence on submarine polarization at depths exceeding a few meters. Our results support this conclusion; there is little or no suggestion of differences in the polarization pattern inside Snell's window compared with the field as a whole. Thus, the patterns of polarization computed by Horváth and Varjú (Horváth and Varjú, 1995) for the situation in shallow water under a flat surface do not appear here. The loss of the atmospheric pattern is certainly due to its destruction by multiple-path scattering; in fact, Waterman (Waterman, 1954) noted that the atmospheric pattern is visible at depths of $2-3 \mathrm{~m}$ in very shallow and clear marine waters.

Finally, polarization in water is surprisingly insensitive to wavelength (see Fig. 2; see also Figs 3-5). Patterns of both the 
overall e-vector orientation and degree of polarization are similar from 360 to $550 \mathrm{~nm}$, although light in the ultraviolet is slightly less polarized than at wavelengths beyond $400 \mathrm{~nm}$. Thus, there is no particular optimum wavelength for photoreceptors that are polarization specialists, but the best signal-to-noise ratios will exist at wavelengths at which light is brightest. So polarization photoreceptors should match the predominant wavelengths of downwelling light, and the middle-wavelength polarization receptors of marine invertebrates are spectrally well placed. The situation with polarization sensitivity is more complicated in fish, in which ultraviolet-sensitive cones are maximally sensitive to vertical e-vector orientation, but green- and red-sensitive cones respond maximally to horizontal e-vectors (Hawryshyn and McFarland, 1987). This implies that visual polarization analysis involves comparisons of different cone mechanisms (Hawryshyn, 1992; Hawryshyn, 2000). Such a system seems non-intuitive and cumbersome, since polarization and spectral variation could be confounded. Nevertheless, if independent visual mechanisms exist that remove spectral brightness variations, the spectral constancy of the polarization pattern does permit different cone classes to contribute to polarization analysis.

To summarize, the patterns of polarization in water that we describe here are spatially robust and relatively insensitive to wavelength. It is now time to extend this work to sample with greater temporal accuracy, over a range of depths and water quality conditions, and with full spherical coverage of the underwater field of partially linearly polarized light.

We thank the staff of the Florida Keys Program, National Undersea Research Center, for much support during the execution of this research. Tim Ford helped prepare the figures. We also thank Justin Marshall for supplying the neutral density filter we used. This work is based on research supported by the National Science Foundation under grant number IBN-9724028 and by the National Undersea Research Center under grant number 9919.

\section{References}

Cronin, T. W., Marshall, N. J. and Caldwell, R. L. (1996). Visual pigment diversity in two genera of mantis shrimps implies rapid evolution. J. Comp. Physiol. A 179, 371-384.

Cronin, T. W., Marshall, N. J. and Caldwell, R. L. (2000). Spectral tuning and the visual ecology of mantis shrimps. Phil. Trans. R. Soc. B 355, 1263-1267.

Cronin, T. W., Marshall, N. J., Caldwell, R. L. and Shashar, N. (1994a). Specialization of retinal function in the compound eyes of mantis shrimps. Vision Res. 34, 2639-2656.

Cronin, T. W., Marshall, N. J., Quinn, C. A. and King, C. A. (1994b), Ultraviolet photoreception in mantis shrimp. Vision Res. 34, 1443-1452.

Goddard, S. M. and Forward, R. B., Jr (1991). The role of the underwater polarized light pattern, in sun compass navigation of the grass shrimp, Palaemonetes vulgaris. J. Comp. Physiol. A 169, 479-491.

Goldsmith, T. H. (1977). Membrane adaptations of visual photoreceptors for the analysis of plane-polarized light. In Research in Photobiology (ed. A. Castellani), pp. 651-658. New York: Plenum Press.

Hawryshyn, C. W. (1992). Polarization vision in fish. Am. Sci. 80, 164-175. Hawryshyn, C. W. (2000). Ultraviolet polarization vision in fishes: possible mechanisms for coding e-vector. Phil. Trans. R. Soc. B 255, 1187-1190.

Hawryshyn, C. W. and Bolger, A. E. (1990). Spatial orientation of trout to partially polarized light. J. Comp. Physiol. A 167, 691-697.

Hawryshyn, C. W. and McFarland, W. N. (1987). Cone photoreceptor mechanisms and the detection of polarized light in fish. J. Comp. Physiol. A 160, 459-465.

Horváth, G. and Varjú, D. (1995). Underwater refraction-polarization patterns of skylight perceived by aquatic animals through Snell's window of the flat water surface. Vision Res. 35, 1651-1666.

Ivanoff, A. and Waterman, T. H. (1958). Factors, mainly depth and wavelength, affecting the degree of underwater light polarization. J. Mar. Res. 16, 283-307.

Jerlov, N. G. (1976). Marine Optics. Amsterdam: Elsevier Scientific.

Lythgoe, J. N. and Hemmings, C. C. (1967). Polarized light and underwater vision. Nature 213, 893-894.

Marshall, N. J., Cronin, T. W. and Shashar, N. (1999). Behavioural evidence for polarisation vision in stomatopods reveals a potential channel for communication. Curr. Biol. 9, 755-758.

Messenger, J. B. (1981). Comparative physiology of vision in molluscs. In Handbook of Sensory Physiology, vol. VII/6C (ed. H. Autrum), pp. 93-200. Berlin: Springer-Verlag.

Novales Flamarique, I. and Hawryshyn, C. W. (1997). Is the use of underwater polarized light by fish restricted to crepuscular time periods? Vision Res. 37, 975-989.

Rossel, S. (1989). Polarization sensitivity in compound eyes. Facets of Vision (ed. D. G. Stavenga and R. C. Hardie), pp. 298-316. Berlin, Heidelberg: Springer-Verlag.

Saidel, W. M., Lettvin, J. Y. and MacNichol, E. F., Jr (1983). Processing of polarized light by squid photoreceptors. Nature 304, 534-536.

Seliger, H. H., Lall, A. B. and Biggley, W. H. (1994). Blue through UV polarization sensitivities in insects. Optimizations for the range of atmospheric polarization conditions. J. Comp. Physiol. A 175, 475-486.

Shashar, N. and Cronin, T. W. (1996). Polarization contrast vision in octopus. J. Exp. Biol. 199, 999-1004.

Shashar, N., Cronin, T. W., Johnson, G. and Wolff, L. B. (1995). Portable imaging polarized light analyzer. Proceedings of the Ninth Meeting on Optical Engineering in Israel. Society of Photo-Optical Instrumentation Engineers 2426, 28-35.

Shashar, N., Hagan, R., Boal, J. G. and Hanlon, R. T. (2000). Cuttlefish use polarization sensitivity in predation on silvery fish. Vision Res. 40, 71-75.

Shashar, N., Hanlon, R. T. and Petz, A. (1998). Polarization vision helps detect transparent prey. Nature 393, 222-223.

Shashar, N., Rutledge, P. and Cronin, T. W. (1996). Polarization vision in cuttlefish: a concealed communication channel? J. Exp. Biol. 199, 2077-2084.

Tyler, J. E. (1963). Estimation of per cent polarization in deep oceanic water. J. Mar. Res. 21, 102-109.

Waterman, T. H. (1954). Polarization patterns in submarine illumination. Science 120, 927-932.

Waterman, T. H. (1981). Polarization sensitivity. In Handbook of Sensory Physiology, vol. VII/6B (ed. H. Autrum), pp. 281-463. Berlin: SpringerVerlag.

Wehner, R. and Rossel, S. (1985). The bee's celestial compass - A case study in behavioral neurobiology. In Experimental Behavior Ecology and Sociobiology (ed. B. Holldobler and M. Lindauer), pp. 11-53. Stuttgart, New York: Fischer Verlag.

Wolff, L. B. and Andreou, A. G. (1995). Polarization camera sensors. Image Vision Comput. 13, 497-510. 